\title{
The role of reflexive thought in the achievement of intercultural competence
}

Reflexivity refers to the capacity for individuals to understand the cultural system and manage their own position within it. Reflexivity is a key concept in the understanding of intercultural communication (UNESCO, 2006; Blasco, 2012), particularly in recognising the ability for individuals to understand and adapt to new cultural contexts. However, the prevailing methods used in intercultural communication (namely that of intercultural competencies), do not place a great emphasis on the role of reflexivity in achieving cultural adaptation. In this paper, I argue for the central positioning of the concept of reflexivity in intercultural education as a mechanism which mediates between intercultural experiences and individual behaviour. I present evidence of the reflexive sequence (subjectobjectsubject) from the reflections of a cohort of students $(n=19)$. Finally, I suggest a pedagogical instrument (a heuristic) for empirically exploring reflexivity in intercultural communication.

Keywords: intercultural education, intercultural communication, intercultural competency, reflexivity, semantic waves

\section{Introduction}

Intercultural contact involves adapting to new contexts with different rules, expectations and obligations. As a consequence, becoming interculturally competent demands reflexive thought, as individuals must confront their new context and figure out an effective course of action. However, without guidance, individuals may find it difficult to find a way through that is effective. Therefore, many organisations and educational institutions have found a need to help students develop habits and values that aid intercultural competence by way of intercultural training. The linguist Michael Byram (1994; 1997) was among the first to try to quantify intercultural competence, developing a set of intercultural competencies linked to pragmatic discourse functions in language assessment - a means of assessment which has been endorsed by the Common European Framework of Reference for Languages. Deardorff (2006), in a survey of influential experts on intercultural communication, found a broad consensus in the use of intercultural competencies in education, observing that individual competencies can be assessed either quantitatively and qualitatively, and that these methods can be creatively applied to different contexts.

Subsequently, intercultural competencies have become an influential part of the methodology and the delivery of teaching and assessment of intercultural communication and there is a general agreement about the methods and assessments used. Intercultural competence is a key concern for a number of businesses, educational institutions and governmental institutions, such as the Higher Education Academy in the UK and UNESCO $(2006,8)$.

However, the concept of intercultural competence is not without criticism. First is that they are necessarily reductionist (Müller-Pelzer, 2011), reducing complex behaviours to acquirable skills, which affects how they are taught. Intercultural competencies present an ideal type of behaviour, which students must perform and evidence as part of a process of assessment. In this way, intercultural competence may be reduced to what the sociologist Basil Bernstein (2000), in his typology of knowledge, refers to as a generic, where knowledge becomes market-oriented, pragmatic and related to the acquisition of skills. These skills are taught in a way which is often stripped of theory, relying on heuristics and tick box approaches to their acquisition, which results in a superficial form of learning. As a consequence, thinking and behaving become a performance as participants aim to show evidence of behaviours which can be documented and used for the purposes of assessment.

For instance, the intercultural competency of tolerance is presented as an ideal behaviour. However, this often does not acknowledge the limits of tolerance, nor may it acknowledge contextual or individual differences (Dasli, 2017). An example of this would be to ask to what extent one should tolerate perceived rudeness in other cultures. Intercultural competencies do not provide a way for 
individuals to draw a line between thinking 'that's just part of their culture' and 'this is not okay'. In order to demonstrate competence, students are invited to display tolerance towards behaviour they may not otherwise be favourable to. Intercultural competencies become somewhat valorised as something that should be adopted uncritically in intercultural communication, rather than as concepts that should be engaged with critically.

Second, since intercultural competencies are reductionist, they deny to some extent the cognitive processes from which intercultural competence emerges, failing to distinguish between the instrumental use of strategies and the process through which these strategies become routine. Intercultural competencies, while providing a broad and varied measure of intercultural competence, do not always adequately reflect the achievement or adoption (or not) of particular behaviours, values or dispositions nor do they allow for inventiveness or rejection of particular competencies. For example, the intercultural competency of empathy is achieved only through experiencing and understanding what somebody else has experienced. Therefore, it involves social relations and reflexive thought, neither of which is adequately captured by way of competencies.

At the heart of this is an epistemological position with an emphasis on individualism (Gershon, 2011). By stressing the instrumental adoption of behavioural strategies, the concept of intercultural competence denies the constraining conditioning effects of social interaction and the associated social and cultural contexts, such as changing power relations or the effects of previous experiences. In some circumstances, individuals may have the freedom to behave how they want to behave and have the self efficacy to choose the skills that they wish to employ. However, in most other circumstances, individuals are strongly guided by cultural and other structural constraints (Caetano, 2015, 68). It is also important to note that while individuals may be able to exercise instrumentality (and though it may make practical sense to do so), this may not necessarily be in ways that will enhance intercultural competence (Blasco, 2012, 484).

Nevertheless, while behaviours and values are socially constructed, individuals are never completely bound by the cultural context. Just because the cultural system valorises particular values and behaviours, it is not a determinist system - the individual has independence within the system, with the agency to make their own meaning of their context, and the ability to develop their own course of action, based on their own personal concerns, even within the tightest of constraints. Individuals are always able to evaluate action (reject, accept or elaborate) in a continuous process of events and reflection as they deliberate on their context in order to find a way through.

I argue that the concept of reflexivity is key to understanding intercultural competence. First, it explains how socialised intercultural habits, values, dispositions and behaviours may emerge from the social context (by way of social interaction); second, it provides an explanation of how individuals may exercise creativity within their contextual constraints (by way of thought); and third it explains how thought may be irrational (by way of cognitive biases). While reflexivity is often included as an intercultural competency itself (equal to other competencies), I argue that reflexive thought is the central generative mechanism behind behavioural change and the achievement of intercultural competence (all behaviours, values, dispositions emerge as a result of reflexive thought). As such, reflexivity should be privileged above other competencies. The concept of reflexivity allows us to separate the individual from the context allowing us to move away from rational models of social behaviour towards a better understanding of how intercultural competence is achieved.

\section{What is Reflexivity?}

The sociologist Margaret Archer $(2003 ; 2007 ; 2012)$ defines reflexivity as "the regular exercise of the mental ability, shared by all people, to consider themselves in relation to their (social) contexts' (Archer, 2007, 4)". Archer conceptualises reflexivity as an internal conversation (2003), where subjects think about the world around them ('what's going on?'), which informs action ('what am I going to do?'). An individual is able to say to themselves about the outside world 'this is right or wrong', which is how we reflexively monitor what we experience (Archer, 2013, 3). 
Reflexivity is not something one has, it is instead something one does as individuals are able to think about their situation and figure things out (Goodson, Hodkinson, Field, Biesta and McLeod, 2011). It is not possible to be unreflexive, as reflexive deliberation is something all humans do, though we may be or more or less constrained in terms of what action we can take depending on the circumstances. When an individual is in a familiar situation and they know what to do, then they do not have to work hard reflexively - they just do it. However, if an individual is an unfamiliar situation (such as intercultural contact), then they must engage in reflexive thought in order to find an effective course of action (Matthews, 2017; 2018). Depending on the context, reflexive thought will result in different courses of action as individuals are constrained or enabled by their circumstances in different ways. Over time, thoughts and action become routine, necessitating a change in intercultural competence. Individuals then feel more or less included in the new context (and the context changes by way of their presence and position within it). However, an individual's thoughts may not necessarily unfurl in a predictable or rational way. That is, we cannot determine how people make sense of their context.

Key to Archer's concept of reflexivity is that the subject (the individual) and object (the context) are analytically separate (what she calls analytical dualism). This means that the individual is conditioned by their social context (by way of social interaction), but also has independence within their context (by way of reflexive thought). In this way, we are able to identify ways in which the individual and the context shape each other. According to Archer, social contexts necessarily precede agency as it is within these contexts that habits, behaviours and values emerge. By placing events and related thoughts on a timeline, Archer (1996) distinguishes between reflection (subject-object) and reflexivity (subject-object-subject), which provides a way of understanding three causally linked stages: the habitualised social routines that individuals bring to the context (the subject), the conditioning powers of interaction with others in the new context (the object), and the subsequent effects on sensemaking by the individual (subject). Some events may reinforce a particular way of thinking or behaviour.

However, other events may compel individuals into new ways of thinking and behaving, which may become habitualised over time. This provides a way of understanding how mindsets and habits change as a result of experiences. By analysing the different strata over time, we are able to separate out the objective features of intercultural contact from the subjective way individuals make sense of their experiences. This distinction allows us to think about intercultural experiences in both concrete terms (by way of description of events) and abstract terms (by way of explanation of events), better explaining how intercultural competence is achieved.

Recent research acknowledges the central role of reflexivity in intercultural education, though there is also acknowledgement that care should be taken regarding its precise effects (Clark and\& Dervin, 2014; Blasco, 2012, Pöllmann, 2016). Blasco (2012) observes how research on the concept of reflexivity in intercultural education emphasises the emancipatory powers of reflexive thought and she cautions against the uncritical application of associated intercultural competences (manifested in terms such as mindfulness or metacognitive capacities). Similarly, Pöllmann (2016) notes how discussion on reflexivity 'fuel unrealistic expectations' on the power of reflexive deliberation in intercultural communication, and he warns against 'uncritical celebrations of reflexivity'. Shim (2015), using a psychoanalytical approach, argues that previous experiences shape how an individual makes sense of current events unconsciously, and thoughts may subsequently lead to uncertainty, ambivalence and irrationality. Zhou and Pilcher (2018), in an analysis of student reflections of intercultural communication, observed that students had a tendency to 'fall back on' essentialist descriptions of culture.

However, no research to date has explored reflexivity as part of the process of intercultural adaptation. The achievement of intercultural competence is a process and understanding the role of reflexive thought is key to understanding how this process works. Reflexive thought is a mechanism that generates new behaviours, values, habits and dispositions. However, we are not endlessly creative and we are strongly shaped by our contexts. Moreover, individuals rely on mental shortcuts informed by previous experiences and unconscious biases. While reflexivity explains the emergence 
of habitualised values and routines, it is not necessarily always benign or rational. There is a need to explore the nature of reflexive thought and its role in the achievement of intercultural competence.

\section{Methods}

In this paper I look at the personal reflections of a cohort of postgraduate international students $(n=19)$ who have undertaken a course on Intercultural Communication at a UK university. Of the 19 students, 18 were Chinese and one was from Saudi Arabia, 15 were female and 4 were male. Such a small sample size is common in educational research, constrained as we are by availability and opportunity. Nevertheless, this sample size provided sufficient for proof-of-concept, though obviously care should be taken when generalising to larger populations. As part of their assessment, students submitted a reflective piece ( 750 words), which asked them to describe their experiences as international students in the UK using concepts learned on the course (students were introduced to around 32 concepts during the course, including the concept of reflexivity). The use of reflective writing to link events, reflection and action is well established in education (see, for example Kolb's Experiential Learning Theory, 1981) and thus written reflections provide a good source of data to explore the mechanism of reflexivity.

The linguist Michael Hoy (2001; 2005) observes how even complex discourse is arranged in simple question-answer patterns (for example, problem-solution or claim-counterclaim). One such pattern is that of abstract-concrete, which has been elaborated by Maton (2013). I analysed these texts for features of reflexive thought using the analytical framework of semantic waves (Maton, 2013). According to Maton, discourse moves from concrete descriptions of the world to abstract explanations and back to concrete descriptions following a wave pattern (what he terms a semantic wave).

First, Maton argues that we describe experiences, events or things in either concrete or abstract terms by moving between strong or weak semantic gravity. Semantic gravity may be understood as the relations between meanings. The stronger the semantic gravity $(\mathrm{SG}+)$ the more that the meaning is context-dependent; the weaker the semantic gravity (SG-), the less meaning is context-dependent. For example, we may refer to culture shock as a way of explaining intercultural contact and the meaning is widely understood (strong semantic gravity). However, the meaning of the similar concept of contextual incongruity is dependent on context, place, location, the shared knowledge of the participants and so on (low semantic gravity). You could not use the same concepts in the same way to different groups of people, because how they would be understood, or whether they would be understood, would depend very much on the context. The movement from concrete (strong semantic gravity) to abstract (weak semantic gravity) over time is an example of weakening semantic gravity.

Second, Maton argues that we then make sense of experiences, events or things by moving between strong or weak semantic density. Semantic density refers to the extent to which meanings are packed or unpacked within a "knowledge practice" - that is, how we attach meaning to events or things (which may be made up of definitions, examples, illustrations, explanations). For Maton, the stronger the semantic density $(\mathrm{SD}+)$, the more meanings are condensed within practices; the weaker the semantic density (SD-), the fewer meanings are condensed. For example, a text may include reference to a particular event (or critical incident) which is explained in terms of examples, definitions, illustrations, building up a constellation of relationships between meanings (low semantic density). However, bringing together explanations, events and examples under an overarching concept such as collectivism-individualism results in high semantic density; the term holds a lot of meaning. A text may describe a particular intercultural event or critical incident, and unpack in terms of concrete meanings (SD-). These meanings would then be packed together and explained in terms of abstract concepts $(\mathrm{SD}+)$.

According to Maton, semantic density and semantic gravity are interdependent. When semantic gravity is low (that is, there are fewer meanings), then semantic density is high (abstract, SD+, SG-), that is, the less meaning given to a concept, then the more the meaning depends on the context (shared knowledge). When semantic density is high (that is, more meaning), then semantic gravity is 
low (concrete, SD-, SG+) - that is, the more meaning attached to a concept, then the less meaning depends on the context. Therefore, the movement between concrete descriptions and abstract explanations over time follows a wave pattern, what Maton calls (a semantic wave). Using this model, it is possible to explore how texts move between concrete and abstract concepts by analysing the language that is used. Concrete descriptions make reference to the world in terms of things such as time, location, participants and other material factors. We might use existential verbs (be, do, have, happen, become), defining relative clauses and adjectives to describe the situation (SG+, SD-). In contrast, the object may be packed up in abstract terms, drawing on theory to explain the context. This may be represented by language such as complex noun phrases, verbs of cause and effect and linking phrases (SG-, SD+).

By imposing Maton's semantic wave on to Archer's reflexive sequence, we can provide both a conceptual and a linguistic framework to analyse reflexive thought related to intercultural events. Students describe their context in concrete terms, then explain these events with reference to abstract concepts and they finally wave down to explain how these events have affected them in concrete terms, providing a way for explaining how events condition individuals over time, mediated by reflexivity. Semantic waves have been used in this research as a measure of reflexive thought, evidencing the process of subject-object-subject deliberation. However, a limitation of this study is that students were writing in their second (or third) language. Although participants were at a B2 level of the Common Europe Framework of Reference (Council of Europe, n.d.) - which specifies that users can understand both concrete and abstract concepts in their second language - non usage of abstract/concrete language in the student reflections may not just be cognitive, but also linguistic.

Submitted student work $(n=19)$ was read, re-read and coded for features of semantic density and semantic gravity and categorised accordingly in relation to Archer's reflexive sequence. This formed the basis for the analysis presented below.

\section{Results}

While the analysis revealed that all reflections could be placed somewhere on the reflexive sequence, they were often not complete. In total, five patterns were discerned from the analysis: subject-only, subject-subject, subject- object, subject-object-subject (not linked) and subject-object-subject (linked). I have inserted the codes - (SD-, SG+) to indicate the concrete and (SD+, SG-) (in bold) to indicate abstract - next to each of the quotes presented here.

\section{a. Subject-only}

The first identifiable pattern is where the student simply described their context. This was characterised by description of the context, but without any relation to how this could be explained in terms of abstract concepts, nor how these experiences affected the subject over time. For example, the following quotes came from one student's reflection, all low semantic density and strong semantic gravity (SD-, SG+, i.e. concrete):

"I am an overseas student from China in the UK." (SD-, SG+)

"Under this situation, I had a huge number of barriers." (SD-, SG+)

"In addition, I suffered abuses [sic] from several British youngsters caused by my nationality." (SD-, SG+)

"I had a huge culture shock." (SD-, SG+)

Although this student had to confront significant challenges, she did not demonstrate any rationalising of her experiences in abstract terms and made no attempt to explain her experiences apart from description, demonstrating passivity towards her situation, a feature of what Archer calls "fractured reflexivity" - a reflexive disposition characterised by an internal conversation which intensifies disorientation (Archer, 2012, 248). Fractured reflexives may tend towards introspection as a 
consequence of the experiences they encounter (turning in on themselves). The link between her experienced events and behaviour was blocked by affect (as a result of her negative experiences) and, subsequently, she was less able to demonstrate understanding of her context and articulate an effective way through.

\section{b. Subject-Subject}

Some students moved from subject-subject, providing a temporal description of change without discussion of how this worked in terms of abstract concepts.

"The eight weeks course has provided me a deeper insight of cultural and intercultural relations than before." (SD-, SG+)

"My views of the world has been expand [sic] by some notions studied and the real life samples provided during class." (SD-, SG+)

"The fact that I am staying in the UK now makes me more aware of the change and the challenge." (SD-, SG+)

"I often felt more comfortable in the Western culture, especially when it comes to individualism, gender equality and the respecting of personal space." (SD-, SG+)

"Now, I learned to understand cross-cultural empathy." (SD-, SG+)

This is an example of intercultural competence as a performance - with the student saying what she believed should be said (that is, that she felt the need to demonstrate personal change as a result of her experiences for assessment purposes), without evidence of critical engagement and only a superficial reference to abstract concepts from the course. She demonstrated an awareness of personal change but did not show evidence of understanding how she got there. Although, she was sincere in her reflections, she did not provide any evidence of a deep understanding of the context.

\section{c. Subject-Object}

Another pattern which emerged from the analysis was a tendency for some students to describe events and explain them using abstract concepts, while not providing an explanation in terms of time. That is, reflections moved from concrete to abstract, but finished there and, as a result, did not provide evidence of understanding how these events conditioned the thinking of the subject. For example:

"At the beginning, I realized that I am the 'new' student to this group" (SD-, SG+)

"I can totally understand why my classmates showed a little distance to me, because of familiarity bias." (SD-, SG+)

"Intergroup bias could explain this situation. In-group means people who share the same things together, out-group means people who do not share same things with them." (SD+, SG)

"Reflexivity means when I found that the situation does not fit me I can make some changes to adapt the new situation quickly." (SD+, SG-)

There is a little more insight here than the previous examples, with the student referring to abstract concepts (intergroup bias) to explain her experiences, though it is somewhat one-dimensional. There is some attempt to link cause and effect ("because of familiarity bias"), but it does not go far enough as an explanation and does not provide an explanation of how this subsequently affected the subject. This indicates that the abstract concepts have not been sufficiently enough internalised for them to have an effect on reflexive thoughts, and as a consequence, are only superficially used to explain the context.

\section{d. Subject-Object-Subject - no links}


Some students described the subject-object-subject following a semantic wave pattern, though they did so as discrete parts with no links between the three stages. This was characterised by referring both to concrete events and abstract concepts, but the links between them were weak. All of the quotes below were from the same student:

"Before arrival in the UK things were familiar to me." (SD-, SG+)

"(On group work) This means I have to attach importance to it via reflexivity, a concept explained as ..." (SD+, SG-)

"Such work can be an effective approach to examining and enhancing my agency." (SD+, SG-)

"... a reflective conversation between classmates and tutors enable me to realise that such cognition appears to be associated with selective perception bias." (SD+, SG-)

"Group work practice supported strengthens my agency via creating my own ideas independently, assigning tasks and transforming ideas." (SD+, SG-)

"I now believe that I become more resilient." (SD-, SG+)

"I am more inclusive and open-minded." (SD-, SG+)

This student treated each stage as distinct items, without linking them. She displayed some reliance on language from input sources (e.g. "strengthens my agency via creating my own ideas independently") and liberal use of intercultural concepts without attributing direct causation onto any of them. Though she demonstrated authenticity in the description of her experiences, and some insight, "such cognition appears to be associated with selective perception bias," it was perhaps a little performative as she tried to cram as many of the concepts into her analysis as possible. She showed evidence of personal change, but this is not fully explained with reference to the abstract concepts. Again, it could be argued that this reflects a performance (for assessment purposes) and does not provide a deep understanding of how she achieved resilience or open-mindedness.

\section{e. Subject-Object-Subject}

Finally, the majority of students were able to explain their experiences in both concrete and abstract terms very well, demonstrating a clear movement from the description of experiences to abstract concepts and back again in a coherent manner. This was characterised by a single focus, event or critical incident, and the selection of one or two concepts to explain these events. For example:

"Before I came to the UK I had been educated in China for about 20 years." (SD-, SG+)

"The classroom teaching is quite country specific." (SD-, SG+)

"In the beginning I was confused." (SD-, SG+)

This student moved to abstraction very clearly by attributing causation to events and explaining it in abstract term:

"Most teachers pay more attention to the result rather than the process. As a result, they prefer to give answers directly." (SD+, SG-)

"The phenomenon is likely to indicate the differences of these two countries' power-distance in educational sector, which means that the power of different groups is unequal." (SD+, SG-)

"A majority of teachers ... are generally not able to be challenged, which lead to a hierarchic distance." (SD+, SG-)

Because this student had clearly understood the context very well, she was able to reflect back on her own context in a critical and authentic way:

"I have changed a lot." (SD-, SG+)

"I still have familiarity bias ..." (SD-, SG+) 
"I have a clearer pursuit of equality and freedom of spirit and consciousness." (SD-, SG+)

Evidence seemed to suggest that students who identified specific personal events seemed better able to rationalise these reflexively. The following student wrote about her experience as an international student in the UK failing a module and subsequently having to retake the course (not the Intercultural Communication course).

"When I came to the UK for further study ..." (SD-, SG+)

"I experienced my first remarkable failure during my life." (SD-, SG+)

"I had really dark days after that failure." (SD-, SG+)

While initially, her internal conversation was not able to find a way through (resulting in the failure of the module), over time reflexive thought did provide a way for her to adapt her course of action successfully.

"I am in a new context now with contextual incongruity, which means that there is somewhat mismatch between China and the UK." (SD+, SG-)

"Lack of cognition about the change of study attitude caused my failure of that exam." (SD+, SG-)

"The failure compelled me into reflexive deliberation, to think how to change to adapt the new teaching environment." (SD+, SG-)

She clearly attributed her negative experience as being due to "lack of cognition" and how reflexive deliberation allowed her to readjust to the new context.

"I become more hard-working and independent." (SD-, SG+)

"My positive transformation also changes others' attitude of my course." (SD-, SG+)

"Failure is not always a bad thing." (SD-, SG+)

Similarly, one student demonstrated the power of reflexive thought on how she dealt with FebruaryMarch 2018 university strike, which had severely impacted her experiences.

"Before I came to the UK, I had not experienced any real strike in China." (SD-, SG+)

She used this experience to engage with the concepts on her course and how it applied to her situation:

"These two opposite choices derive from different conditions of power distribution in the two societies." (SD+, SG-)

"... the concept of power-distance, which demonstrates the extent of acceptance of unequal power distribution." (SD+, SG-)

"As a result of this different power distributions ..." (SD+, SG-)

"These different choices are consistent with one of Hofstede's cultural dimensions (Uncertainty Avoidance)." (SD+, SG-)

"This is an adaptive process explained effectively by the ABC model." (SD+, SG-)

An understanding of the context meant that she was able to find room for reflexive manoeuvre within her constraints, which had a real effect on her changing habits and attitudes.

"I began to think about what I can to minimise the negative influence on my study." (SD-, SG+)

"I realised I should consciously adjust my attitude towards study." (SD-, SG+)

These examples show how subjects adapted their behaviour to their context, by way of reflexive thought. In some cases, the evidence shows that reflexivity may not always lead to an effective course 
of action (the reflexive sequence is interrupted by affect or other factors). Theories of intercultural contact and intercultural competencies were very useful as guides for the students to structure their deliberations and were used to rationalise and justify changes in behaviour or values.

\section{Discussion}

Reflexive thought has been identified as a generative mechanism which links external experienced events to internalised habits, routines and values. It provides an explanation of how intercultural experiences compel individuals into thought, which forms the basis of new socialised habits, routines and values. However, reflexive thought does not always benignly result in intercultural competence, and students still need guidance. Intercultural competencies can provide useful heuristic devices in the development of an intercultural mindset, but the tendency to rely on instrumentality to explain behaviour means that intercultural competence may be reduced to tick-box approaches to learning, which does not adequately reflect behavioural change.

This research is important in that it shows how intercultural competence was achieved, by a process of experience of events, thoughts and action. Students deliberated on their experiences and, strongly guided by intercultural theories studied during the module, subsequently adopted behaviours or values more congruent with the new context. Intercultural concepts provided useful conceptual tools in order to aid intercultural competence, but it was the student that found their own way through. However, intercultural competence is not smooth or predictable. Reflexivity may be interrupted in some way, by affect or other events. Exploring reflexivity in this way means that teachers' institutions can identify instances where an effective course of action may be blocked.

This research also shows that there is a tendency towards consensus in the achievement of intercultural competence. It is a human psychological trait that individuals must seek consensus in social groups in order to make it easier to perform social tasks (Lieberman, 2013). This is done by intuiting what should be done through observation. Every social interaction requires this intuition, so it has a reciprocal effect. Consequently, there may emerge a shared cognition from people sharing experiences, a tendency for values, behaviours and habits to converge over time - what may become an intercultural mindset. This research shows how students who found an effective way through were able to intuit the appropriate actions to conform, and all tended towards similar behaviours and ways of thinking. Although reflexive thought mediated experiences and action, students were still required to use their intuition as a guide. Since the complexity of their experiences were sometimes overwhelming, intercultural competencies provided effective heuristics in informing intuition and deliberation.

\section{Pedagogical Implications}

The tendency for intercultural education towards instrumental explanations of behaviour can be approached in two ways - either by developing new competencies, or by improving the models that are already widely used. Tempting though it is to dispose of over 40 years of research on intercultural education, since intercultural competencies seem to share a tendency towards performativity, it is perhaps a more elegant solution to identify and improve on the limitations of these models. Reflexivity provides a way of explaining how competencies may emerge from experience. In this way, we can see that events necessarily precede action, and action is mediated by reflexive deliberation, which can be captured through relating concrete descriptions of events to abstract explanations of these events, and how they influence behaviour:

\section{[Figure 1 near here]}

By combining Archer's reflexive sequence with Maton's semantic wave, this simple heuristic (rule-ofthumb) can be used to enhance existing intercultural competencies by adding a temporal dimension. By providing an explanation of events, thoughts and habits over time, it is possible to see how events condition behaviour. The application of this simple heuristic to any critical incident or intercultural competency can aid students in their reflections. Students may describe a particular event (what 
happened, who was involved), then refer to intercultural concepts to explain those events (such as power-distance), then finally reflect on how they negotiated the event. Prompts for context, explanations and language stimulate deliberation, and help students make links between intercultural events and how it influences them.

Finally, in order to develop models of practice that can be generalised to wider student populations, more research needs to be done in a range of contexts. In particular, the presence (or not) of fractured reflexivity is lacking in this research, and there is a need to be able to explore the experiences of students who find themselves constrained in the new environment and unable to find an effective way through.

\section{References}

Archer, M. S. 1996. Culture and agency: The place of culture in social theory. Cambridge University Press.

Archer, M. S. 2003. Structure, agency and the internal conversation. Cambridge University Press.

Archer, M. S. 2007. Making our way through the world: Human reflexivity and social mobility. Cambridge University Press.

Archer, M. S. 2012. The reflexive imperative in late modernity. Cambridge University Press.

Archer, M. S. 2013. Reflexivity. Sociopedia.isa.

http://www.sagepub.net/isa/resources/pdf/Reflexivity2013.pdf

Bernstein, B. B. 2000. Pedagogy, symbolic control, and identity: Theory, research, critique. (No. 4). Rowman \& Littlefield.

Blasco, M. 2012. "On reflection: is reflexivity necessarily beneficial in intercultural education?" Intercultural Education 23 (6): 475-489.

Byram, M. 1994. Teaching-and-learning language-and-culture (Vol. 100). Multilingual matters.

Byram, M. 1997. Teaching and assessing intercultural communicative competence. Multilingual Matters.

Clark, J. S. B., \& Dervin, F. (Eds.). 2014. Reflexivity in language and intercultural education: Rethinking multilingualism and interculturality (Vol. 2). Routledge.

Council of Europe, n.d. Common European Framework of Reference for Languages

(CEFR): Global Scales. [online] https://www.coe.int/en/web/common-europeanframeworkreference-languages/table-1-cefr-3.3-common-reference-levels-global-scale

Dasli, M. 2017. "Intercultural education of tolerance and hospitality." Discourse: Studies in the Cultural Politics of Education 38 (5): 676-687.

Gershon, I. 2011. "Neoliberal agency." Current Anthropology, 52 (4): 537-555.

Goodson, I. F., Hodkinson, P., Field, J., Biesta, G., \& Macleod, F. J. 2011. Improving learning through the lifecourse: Learning lives. Routledge.

Hoey, M., 2001. Textual interaction: An introduction to written discourse analysis. Routledge.

Hoey, M. 2005. Lexical priming: a new theory of language and words. Routledge.

Kolb, D. A. 1981. "Experiential learning theory and the learning style inventory: a reply to Freedman and Stumpf." Academy of Management Review 6 (2): 289-296.

Lieberman, M. 2013. Social: why our brains are wired to connect. Oxford University Press. 
Maton, K. 2013. "Making semantic waves: A key to cumulative knowledge-building." Linguistics and Education 24 (1): 8-22.

Matthews, B. 2017. "I wouldn't imagine having to go through all this and still be the same person. No way': Structure, Reflexivity and International Students." Journal of Research in International Education 16 (3): 265-278.

Matthews, B. 2018. "'You Just Use your Imagination and Try to Fix it': Agential Change and International Students." Journal of International Students 8 (1): 332-350.

Müller-Peltzer, W. 2011. "Intercultural Competence evaluated - the End of an Illusion." Proceedings INTED 2011 Valencia.

http://www.wirtschaft.fhdortmund.de/ ib/DIfEalS/HTML/PDF/impect5_08_mp_intercultural_comp _evaluated.pdf

Odağ, Ö., Wallin, H. R., \& Kedzior, K. K. 2016. "Definition of intercultural competence according to undergraduate students at an international university in Germany." Journal of Studies in International Education 20 (2): 118-139.

Pöllmann, A. 2016. "Habitus, reflexivity, and the realization of intercultural capital: The (unfulfilled) potential of intercultural education." Cogent Social Sciences 2.

Shim, J. M. 2015. "Transference, Counter-transference, and Reflexivity in Intercultural Education." Educational Philosophy and Theory, 47 (7): 675-687.

UNESCO. 2006. UNESCO Guidelines on Intercultural Education. Paris: UNESCO.

Wang, Y. A., \& Kulich, S. J. 2015. "Does context count? Developing and assessing intercultural competence through an interview-and model-based domestic course design in China." International Journal of Intercultural Relations 48: 38-57.

Zhou, V. W., \& Pilcher, N. 2018. "'Intercultural competence' as an intersubjective process: a reply to 'essentialism.'” Language and Intercultural Communication 18 (1): 